\title{
Rotor Position Estimation with Hall-Effect Sensors in Bearingless Drives
}

\author{
Patricio Peralta, Jacopo Leo, Yves Perriard \\ Laboratory of Integrated Actuators, École polytechnique fédérale de Lausanne \\ Neuchâtel, Switzerland \\ Email: \{juan.peraltafierro, jacopo.leo, yves.perriard\}@epfl.ch \\ URL: https://lai.epfl.ch/
}

Keywords

$\ll$ Magnetic bearings $\gg, \ll$ Sensor $\gg, \ll$ Permanent magnet motor $\gg, \ll$ Motion Control $\gg$

\begin{abstract}
The estimation of rotor position is fundamental for the commissioning of magnetically-levitated drives. The sensors required for this measurement must be close to the rotor, often requiring constructive measures that may limit the application potential of a drive. This publication presents the estimation of rotor angle and position, based on Hall-effect sensors installed on a slotless bearingless drive. This scheme enables sensor mounting away from the rotor, thus simplifying its encapsulation.
\end{abstract}

\section{Introduction}

Contemporary research trends strive towards the miniaturization of electrical drives, which has lead to increased investigation efforts towards high-speed permanent magnet motors [1]. Nonetheless, the classical ball-bearing approach to this venture is defiant [2], as rotordynamical effects [3] and mechanical losses [4] stand in the way of reliable, high-speed operation. Contactless magnetic bearings enable getting past these issues. They are reliable and have successfully reached speeds over $10 \mathrm{krpm}$ in different sizes and topologies $[5,6,7]$.

Successful levitation needs information about the rotor angle in order to generate bearing forces in the desired direction [8]. Rotor angle is classically estimated by measuring the magnetic fields with Halleffect sensors; yet these fields also contain information about the rotor position in the airgap that usually goes unexploited [9].

The information about rotor position is usually estimated by additional sensors. To measure it, capacitive, optical, and eddy current sensors are among the preferred sensor approaches [8]. They usually have to be placed inside the airgap, often requiring special constructive means that may damper the application range of the drive $[10,11]$. This paper validates how only Hall-effect sensors, distributed as in Fig. 1a, enable the estimation of rotor position and angle.

Indeed, bearingless motors with a slotted stator have already been comissioned only by measuring the magnetic induction hall-effect sensors [12], but its specifics are not disclosed. In this paper, the principles for measuring the $x-y$ position, angle, and additionally axial rotor position of a one pole-pair rotor with a slotless stator are presented. A mathematical model is inspired by computational Finite Element (FE) method simulations, and its validity is experimentally tested. Exploiting the mathematical model of the magnetic fields, a model for rotor position estimation is developed.

The proposed sensor array can lay outside the airgap, enabling the construction of simple encapsulation concepts. Conveniently enough, stray fields outside the airgap show low intensities, compatible to offthe-shelf ratiometric Hall-effect sensors. 


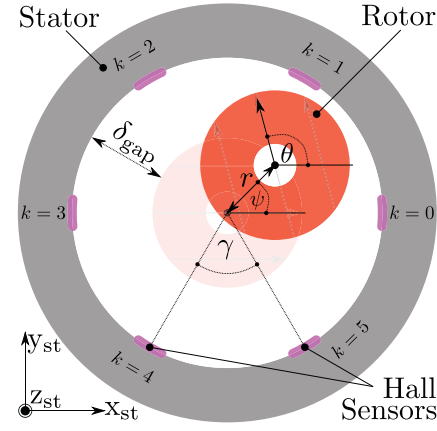

(a)

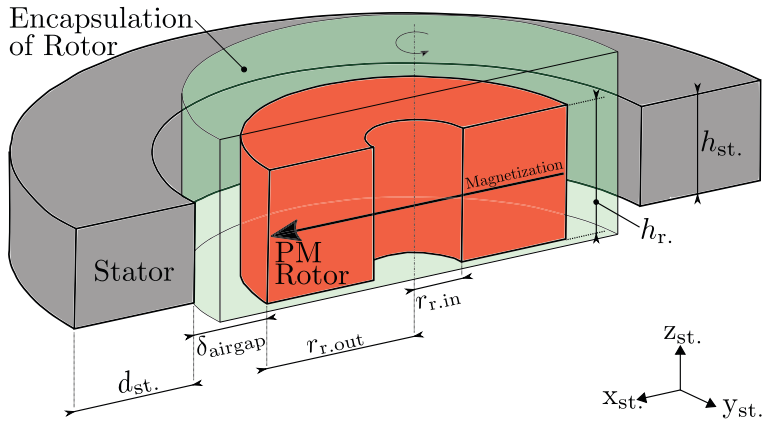

(b)

Fig. 1: (a) Top view of a drive with an exaggeratedly uncentered rotor and its symmetrical positioning of 6 Hall-effect sensors at the stator. Its radial position and magnetic orientation are determined by coordinates $(r, \psi)$ and $\theta$, respectively. (b) Cross section of a slotless bearingless disc drive, studied in this paper, with an encapsulated rotor and its relevant dimensions.

The mathematical derivation of angle and position estimation are general for any one pole-pair magnetically levitated (mag-lev) drives. Nonetheless, this paper focuses on its implementation and validation in a disc drive (or slice motor) [13], as shown in Fig. 1b. Among magnetic bearing topologies, their geometric properties (namely the large diameter-to-length ratio) offer axial and tilting passive stability thus simplifying the overall drive system and its control.

\section{Mathematical Modelling}

\section{Field in the Airgap}

A one pole-pair, diametrically-magnetized permanent magnet (PM) rotor inside a slotless stator is studied. Figure 2a depicts its cross section, with a centric and eccentric rotor along its FE-simulated radial magnetic induction. Rotor eccentricity impacts the magnetic field distribution [14].

The radial magnetic induction is sensed at two positions ( 0 and $\pi / 2 \mathrm{rad})$ for this example. Each sensor has a local coordinate system, with normal and tangential directions denominated with $(n, \tau)$ respectively.

Figure $2 b$ depicts the magnetic induction of the rotating rotor measured by both sensors. The magnetic induction of increasing eccentricity $r / \delta_{\text {gap }}$ is depicted in darkening colors.

When the rotor moves parallel to a normal sensor direction, i.e. parallel to $n_{0}$, the amplitude of the magnetic induction measured by the sensor increases with rotor eccentricity. On the other hand, a movement parallel to the tangential sensor direction $\tau_{\pi / 2}$ changes the phase of the measured magnetic induction.

For the a non-turning rotor, eccentricity is measured as a local change in measured magnetic induction, as shown in Fig. 2c . For small displacements, this alterations in the radial magnetic induction changes are practically linear to rotor displacement, as plotted in the Figure. These insights are implemented in the modelling of the magnetic induction in the airgap and its dependency with rotor displacement.

The motor magnetic fields are now studied by an array of six Hall-effect sensors $k=0,1, \ldots, 5$. They are symmetrically distributed on the interior of the stator, with separation $\gamma=\pi / 3$, distributed as in Fig. 1a. Thus, each sensor $k$ measures a phase-shifted magnetic flux density $B_{k}$ as in

$$
\begin{aligned}
B_{k}(\theta, r, \psi, k) & =\hat{B}(r, \psi, k) \cdot \cos \left(\theta-k \cdot \gamma+\tau_{k}\right), \\
\text { with } k & =0,1, \ldots, 5, \text { and } \gamma=\pi / 3,
\end{aligned}
$$

with $\tau_{k}$ modelled as the product of rotor displacement projected onto the tangential axis of the coordinate system of the $k$ th-sensor $r_{\tau, k}$ and $s_{\tau}$ the phase-shift to displacement sensibility as in $\tau_{k}=s_{\tau} \cdot r_{\tau, k}$. This proportional phase-shift is hereafter neglected, as it is non-linear and theoretically distorts measurements only at large rotor deflections. 


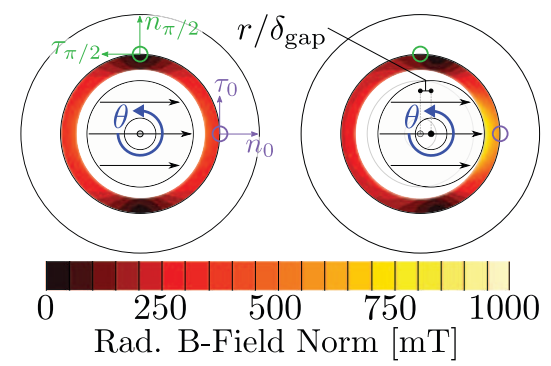

(a)

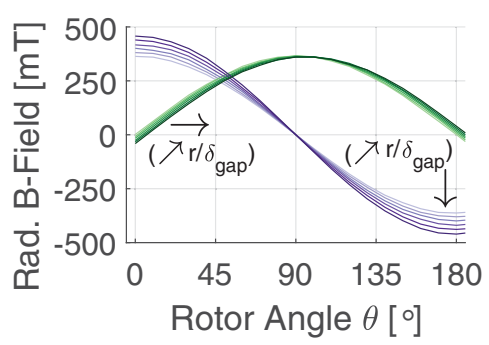

(b)

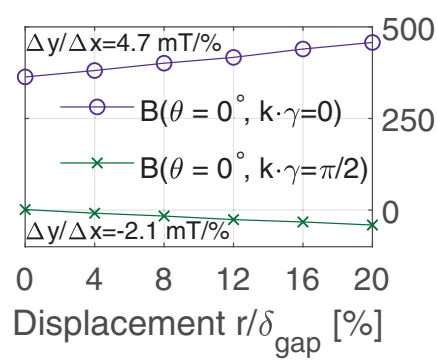

(c)

Fig. 2: (a) The one-pole pair, radial magnetic induction distribution is distorted when the rotor is eccentric. (b) In the eccentricity direction (at purple $\bigcirc$ ), this increases the amplitude of the radial magnetic induction. Perpendicular to the eccentricity (at green $\bigcirc$ ), it affects the phase of the radial magnetic induction. Darker colors represent a larger $r / \delta_{\text {gap }}$ eccentricity. (c) The latter effects have a linear impact upon the radial magnetic induction sensed by the sensors.

For feeble rotor displacements $r$, the peak magnetic flux density $\hat{B}(r, \psi, k)$ of Eq. (1) varies as in [9]

$$
\begin{aligned}
\hat{B}(r, \psi, k)=\hat{B}_{0} \cdot \frac{1}{1-r / \delta_{\text {gap }} \cdot \cos (\psi-k \cdot \gamma)} & \approx \hat{B}_{0} \cdot\left[1+r / \delta_{\text {gap }} \cdot \cos (\psi-k \cdot \gamma)\right], \\
& =\hat{B}_{0} \cdot\left[1+r \cdot s_{r} \cdot \cos (\psi-k \cdot \gamma)\right),
\end{aligned}
$$

using the Taylor series approximation of $1 /(1-x) \approx(1+x)$, for $|x|<1$. The normal flux path is initially assumed to be determined by $\delta_{\text {gap }}$, which appears as gain $1 / \delta_{\text {gap }}$ of radial displacement $r$. Yet, this flux path is not always straight nor evident, specially in disc drives. Therefore, the equation is further generalized with a radial displacement gain $s_{r}$ that depends on where the sensor is placed.

Hall-effect sensors also sense the axial displacements (z-direction) of the rotor. If they are not placed at the same axial plane as the rotor, they can sense if this moves away or approaches. This might prove useful in applications where axial vibrations need to be suppressed [15]. Provided a small displacement, the change in peak magnetic flux density $\hat{B}$ can also modelled in a linear manner as in Eq. 2.

Considering Eqs. (1) - (2) and the possibility of axial displacement, the magnetic field density that each Hall-effect sensor $k$ measures is

$$
B_{k}^{ \pm}(\theta, r, \psi, k, z)=\hat{B}_{0} \cdot \underbrace{\left[1+r \cdot s_{r} \cdot \cos (\psi-k \cdot \gamma)\right]}_{\text {polar rotor displacement }} \cdot \underbrace{\left(1 \pm z \cdot s_{z}\right)}_{\text {axial rotor displacement }} \cdot \underbrace{\cos (\theta-k \cdot \gamma)}_{\text {rotor rotation }}
$$

with the + or - sign for axial rotor displacement if the Hall-effect sensor lies on over or under the rotor still stand position, respectively.

A symmetric array of 12 Hall-effect sensors ( 6 top and 6 bottom) is employed, so as to measure rotor angle, its radial position, and its axial position.

\section{Model for the Estimation of Rotor Angle}

The objective of rotor angle $\theta$ estimation is to generate a signal pair $(\cos \theta, \sin \theta)$ out of Hall-effect sensor measurements. For this, the effects of radial and axial displacement contained in each Hall-effect sensor measurement in Eq. (3) are to be eliminated.

The effect of axial displacement are eliminated by adding the respective top and bottom sensors; that is, for each top-bottom Hall-effect sensor pair $k=0,1, \ldots, 5$,

$$
\begin{aligned}
B_{k}^{\operatorname{sum}}(\theta, r, \psi, k) & =B_{k}^{+}(\theta, r, \psi, k, z)+B_{k}^{-}(\theta, r, \psi, k, z) \\
& =2 \cdot \hat{B}_{0} \cdot\left[1+r \cdot s_{r} \cdot \cos (\psi-k \cdot \gamma)\right] \cdot \cos (\theta-k \cdot \gamma) .
\end{aligned}
$$

To cancel the effect of the displaced rotor in $r \cdot s_{r}$, all opposing Hall-sensor pairs signals $B_{k}^{\text {sum }}$ for $k=$ 
$0,1,2$ are subtracted, resulting in

$$
\begin{aligned}
B_{k}^{\text {sum }} & (\theta, r, \psi, k)-B_{k+3}^{\text {sum }}(\theta, r, \psi, k+3) \\
= & \left\{2 \cdot \hat{B}_{0} \cdot\left[1+r \cdot s_{r} \cdot \cos (\psi-k \cdot \gamma)\right] \cdot \cos (\theta-k \cdot \gamma)\right\} \\
& -\left\{2 \cdot \hat{B}_{0} \cdot\left[1+r \cdot s_{r} \cdot \cos (\psi-k \cdot \gamma-3 \cdot \gamma)\right] \cdot \cos (\theta-k \cdot \gamma-3 \cdot \gamma)\right\} \\
= & 4 \cdot \hat{B}_{0} \cdot \cos (\theta-k \cdot \gamma),
\end{aligned}
$$

since $3 \cdot \gamma=\pi$. These signals are combined and $(\cos \theta, \sin \theta)$ is obtained as

$$
\begin{aligned}
S_{\cos }= & \left\{B_{0}^{\text {sum }}(\theta, r, \psi, 0)-B_{3}^{\text {sum }}(\theta, r, \psi, 3)\right\}+1 / 2 \cdot\left\{B_{1}^{\text {sum }}(\theta, r, \psi, 1)-B_{4}^{\text {sum }}(\theta, r, \psi, 4)\right\} \\
& -1 / 2 \cdot\left\{B_{2}^{\text {sum }}(\theta, r, \psi, 2)-B_{5}^{\text {sum }}(\theta, r, \psi, 5)\right\} \\
= & 6 \cdot \hat{B}_{0} \cdot \cos \theta, \\
S_{\text {sin }}= & \sqrt{3} / 2 \cdot\left\{B_{1}^{\text {sum }}(\theta, r, \psi, 1)-B_{4}^{\text {sum }}(\theta, r, \psi, 4)\right\}+\sqrt{3} / 2 \cdot\left\{B_{2}^{\text {sum }}(\theta, r, \psi, 2)-B_{5}^{\text {sum }}(\theta, r, \psi, 5)\right\} \\
= & 6 \cdot \hat{B}_{0} \cdot \sin \theta .
\end{aligned}
$$

Ultimately, the peak magnetic induction $\hat{B}_{0}$ can also be estimated from these signals as in

$$
\hat{B}_{0}=1 / 6 \cdot \sqrt{S_{\mathrm{cos}}^{2}+S_{\text {sin }}^{2}}
$$

\section{Model for the Estimation of Rotor Radial Position}

Rotor position information on the $x-y$ plane is calculated analogous to Eq. (5) by adding opposing sensors for $k=0,1,2$ as

$$
B_{k}^{\text {sum }}(\theta, r, \psi, k)+B_{k+3}^{\text {sum }}(\theta, r, \psi, k+3)=4 \cdot \hat{B}_{0} \cdot\left[r \cdot s_{r} \cdot \cos (\psi-k \cdot \gamma)\right] \cdot \cos (\theta-k \cdot \gamma),
$$

thus obtaining signals which are proportional to polar displacement $r$. Once again, analogous to Eq. (6), two perpendicular signals can be obtained by combining sensor information as in

$$
\begin{aligned}
S_{\mathrm{X}_{\mathrm{rot}}=} & \quad\left\{B_{0}^{\text {sum }}(\theta, r, \psi, 0)+B_{3}^{\text {sum }}(\theta, r, \psi, 3)\right\}-1 / 2 \cdot\left\{B_{1}^{\text {sum }}(\theta, r, \psi, 1)+B_{4}^{\text {sum }}(\theta, r, \psi, 4)\right\} \\
& -1 / 2 \cdot\left\{B_{2}^{\text {sum }}(\theta, r, \psi, 2)+B_{5}^{\text {sum }}(\theta, r, \psi, 5)\right\} \\
= & 3 \cdot \hat{B}_{0} \cdot\left(r \cdot s_{r}\right) \cdot \cos (\theta+\psi), \\
S_{\mathrm{Y}_{\mathrm{rot}}}= & \sqrt{3} / 2 \cdot\left\{B_{1}^{\text {sum }}(\theta, r, \psi, 1)+B_{4}^{\text {sum }}(\theta, r, \psi, 4)\right\}-\sqrt{3} / 2 \cdot\left\{B_{2}^{\text {sum }}(\theta, r, \psi, 2)+B_{5}^{\text {sum }}(\theta, r, \psi, 5)\right\} \\
= & 3 \cdot \hat{B}_{0} \cdot\left(r \cdot s_{r}\right) \cdot \sin (\theta+\psi),
\end{aligned}
$$

which are the $x$ and $y$ coordinates of the rotor in the rotating coordinate system of the rotor, i.e. it depends on $\theta$. To control the levitation, the position on the fixed coordinate system, i.e. not $\theta$ dependent, are essential [8]. This is easily obtained by taking advantage from the results of Eq. (6) and rotating $\left(S_{\mathrm{X}_{\text {rot }}}, S_{\mathrm{Y}_{\text {rot }}}\right)$ by computing

$$
\left[\begin{array}{l}
S_{\mathrm{X}_{\mathrm{st}}} \\
S_{\mathrm{Y}_{\mathrm{st}}}
\end{array}\right]=\frac{2}{S_{\mathrm{cos}}^{2}+S_{\mathrm{sin}}^{2}} \cdot\left[\begin{array}{cc}
S_{\mathrm{cos}} & S_{\mathrm{sin}} \\
-S_{\mathrm{sin}} & S_{\mathrm{cos}}
\end{array}\right] \cdot\left[\begin{array}{l}
S_{\mathrm{X}_{\mathrm{rot}}} \\
S_{\mathrm{Y}_{\mathrm{rot}}}
\end{array}\right]=\left(r \cdot s_{r}\right) \cdot\left[\begin{array}{c}
\cos \psi \\
\sin \psi
\end{array}\right],
$$

hence obtaining two signals projected on the fixed coordinate system. They are directly proportional to the displacement $r$ and independent from $\hat{B}_{0}$. 


\section{Position Error of Estimation due to Phase-Shift}

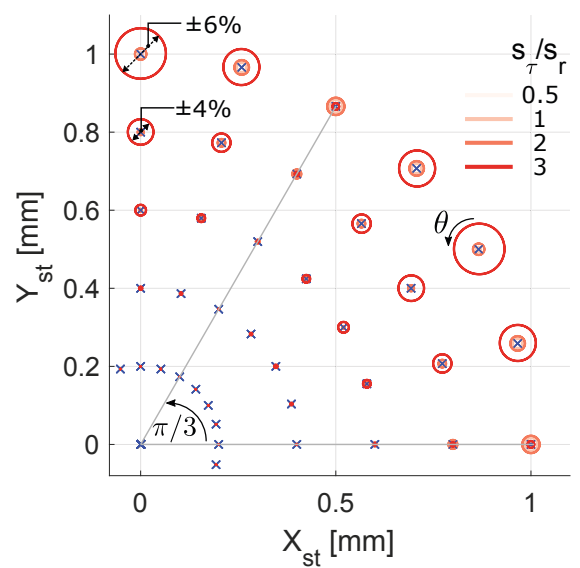

Fig. 3: Actual vs. estimated rotor position, in $\times$ and $\bigcirc$ respectively. The other position quadrants are symmetric.
The position estimation scheme is developed by neglecting the phase-shift $\tau_{k}$ of Eq. (1), generated by rotor displacement. This allows for a linear model and a more intuitive derivation.

Phase-shift $\tau_{k}$ has yet an influence upon the fidelity of position estimation. This becomes apparent for increasing rotor displacements $r$ and phase-shift to displacement sensibility $s_{\tau}$. Figure 3 depicts the true vs. model-estimated position of the rotor for increasing sensibilities $s_{\tau}$. As the rotor turns, the estimated position circles around the true position. This behaviour is not foreseen by the estimator.

Yet, the error radius is minimized when the rotor is displaced towards a sensors. This would speak for setups with more sensors around the airgap for high-precision position estimation. Nevertheless, the depicted $s_{\tau} / s_{r}$ values are already very large with respect to values obtained by FE simulations. According to them, rather small sensitivity values $s_{\tau} / s_{r}<1$ are expected. Therefore, position estimation error should be rather small.

\section{Model for the Estimation of Rotor Axial Position}

If only top or bottom sensors were available, signals $\left(S_{\mathrm{cos}}, S_{\mathrm{sin}}\right)$ and $\left(S_{\mathrm{X}_{\mathrm{st}}}, S_{\mathrm{Y}_{\mathrm{st}}}\right)$ would remain modulated by factor $\left(1 \pm z \cdot s_{z}\right)$. Axial position information could be estimated out of the angle signals that would constantly carry an offset. Yet having top and bottom sensors enables finding a tidier expression for axial displacement $z$, by calculating the difference of $(\cos \theta, \sin \theta)$ signals of the upper and lower sensors,

$$
\begin{aligned}
S_{\mathrm{cos}}^{ \pm}= & \left\{B_{0}^{ \pm}(\theta, r, \psi, 0)-B_{3}^{ \pm}(\theta, r, \psi, 3)\right\}+1 / 2 \cdot\left\{B_{1}^{ \pm}(\theta, r, \psi, 1)-B_{4}^{ \pm}(\theta, r, \psi, 4)\right\} \\
& -1 / 2 \cdot\left\{B_{2}^{ \pm}(\theta, r, \psi, 2)-B_{5}^{ \pm}(\theta, r, \psi, 5)\right\}=6 \cdot \hat{B}_{0} \cdot \cos \theta \cdot\left(1 \pm z \cdot s_{z}\right) \\
\rightarrow S_{z-\cos }= & S_{\cos }^{+}-S_{\cos }^{-}=6 \cdot \hat{B}_{0} \cdot \cos \theta \cdot z \cdot s_{z}, \\
S_{\sin }^{ \pm}= & \sqrt{3} / 2 \cdot\left\{B_{1}^{ \pm}(\theta, r, \psi, 1)-B_{4}^{ \pm}(\theta, r, \psi, 4)\right\}+\sqrt{3} / 2 \cdot\left\{B_{2}^{ \pm}(\theta, r, \psi, 2)-B_{5}^{ \pm}(\theta, r, \psi, 5)\right\} \\
= & 6 \cdot \hat{B}_{0} \cdot \sin \theta \cdot\left(1 \pm z \cdot s_{z}\right) \\
\rightarrow S_{z-\sin }= & S_{\sin }^{+}-S_{\sin }^{-}=6 \cdot \hat{B}_{0} \cdot \sin \theta \cdot z \cdot s_{z},
\end{aligned}
$$

which means that a signal proportional to $z$ and then normalized by $1 /\left(S_{\cos }^{2}+S_{\sin }^{2}\right)=1 /\left(36 \cdot \hat{B}_{0}^{2}\right)$ can be obtained as in

$$
D_{z}=\left(S_{z-\cos } \cdot S_{\cos }+S_{z-\sin } \cdot S_{\sin }\right) /\left(S_{\cos }^{2}+S_{\sin }^{2}\right)=z \cdot s_{z}
$$

\section{Test Bench and Hall-Effect Sensor Mounting}

The presented mathematical model is validated on the experimental test bench of Fig. 4. The disc drive of Fig. $1 \mathrm{~b}$ is fabricated with the materials and dimensions of Table I and mounted.

Commercially-available, ratiometric Hall-effect sensors from Texas Instruments DRV5055 series [16] are contemplated, given their simple 3-pad implementation. They are available in TO-92 and SOT-23 packaging, suitable for radial and axial PCB mounting, respectively.

These Hall-effect sensors are mounted onto the fabricated PCBs of Fig. 5a. Two PCBs, one below and above the stator, help to spatially distribute the sensors as in Fig. 1a. The PCBs enable the two different DRV5055 mounting options shown in Fig. 5b. 
Table I: Materials and dimensions of mounted motor.

\begin{tabular}{lc}
\hline \hline Rotor Mat. & NdFeB42H [17] \\
Stator Mat. & Metglas 2605SA1 [18] \\
\hline$d_{\text {st. }}$ & $8 \mathrm{~mm}$ \\
$\delta_{\text {airgap. }}$ & $5 \mathrm{~mm}$ \\
$r_{\text {r.out }}$ & $10 \mathrm{~mm}$ \\
$r_{\text {r.in }}$ & $3 \mathrm{~mm}$ \\
$h_{\mathrm{r}}$ & $8 \mathrm{~mm}$ \\
$h_{\text {st. }}$ & $6 \mathrm{~mm}$ \\
\hline \hline
\end{tabular}

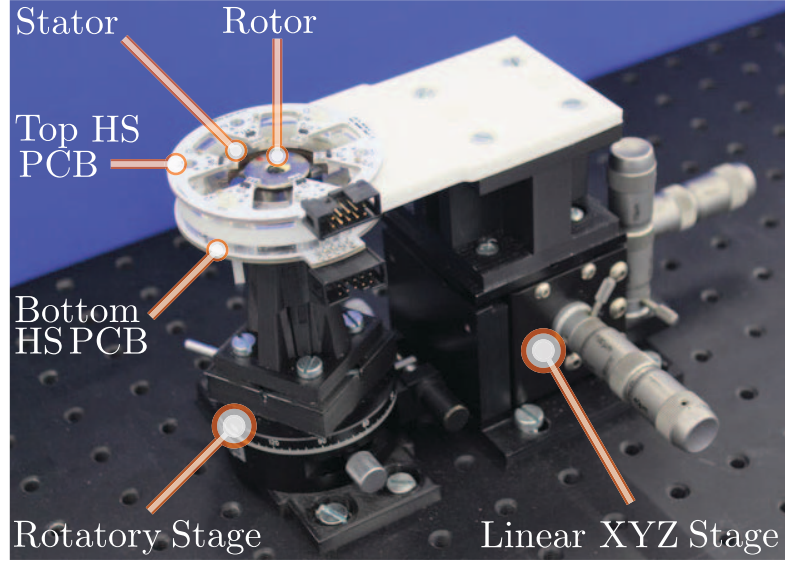

Fig. 4: Model validation test bench. The PM rotor is fixed to a rotatory stage for $\theta$, whereas the ferromagnetic stator and and PCBs are fixed on a XYZ stage.

\section{Model Validation}

The outputs of the 12 hall-effect sensors are installed onto 12-bit ADCs. A displacement is manually imposed onto the screws of the linear or rotational stage, and the data is then logged in.

For comparison purposes, the measurements are obtained once using radial-field measuring sensors, and then axial-field measuring sensors, mounted as in Fig. 5b. For the rest of the paper, these are referred according to the orientation of the magnetic induction they measure, as radial and axial respectively. The results and their performance are then compared.

\section{Hall-Effect Sensor Values}

Figure 6a depicts the mean values of the bottom and top sensors when the rotor is turned in the center of the airgap. Given the proposed installation, axial sensors measure larger sensor amplitudes. This enhances signal-to-noise ratio, rendering axial orientation preferable to the radial for angle estimation.

The signals are combined to generate $(\cos \theta, \sin \theta)$. Despite its lower amplitude of the radially-oriented sensors, both orientation draw a linear relationship with the angle imposed on the test bench.
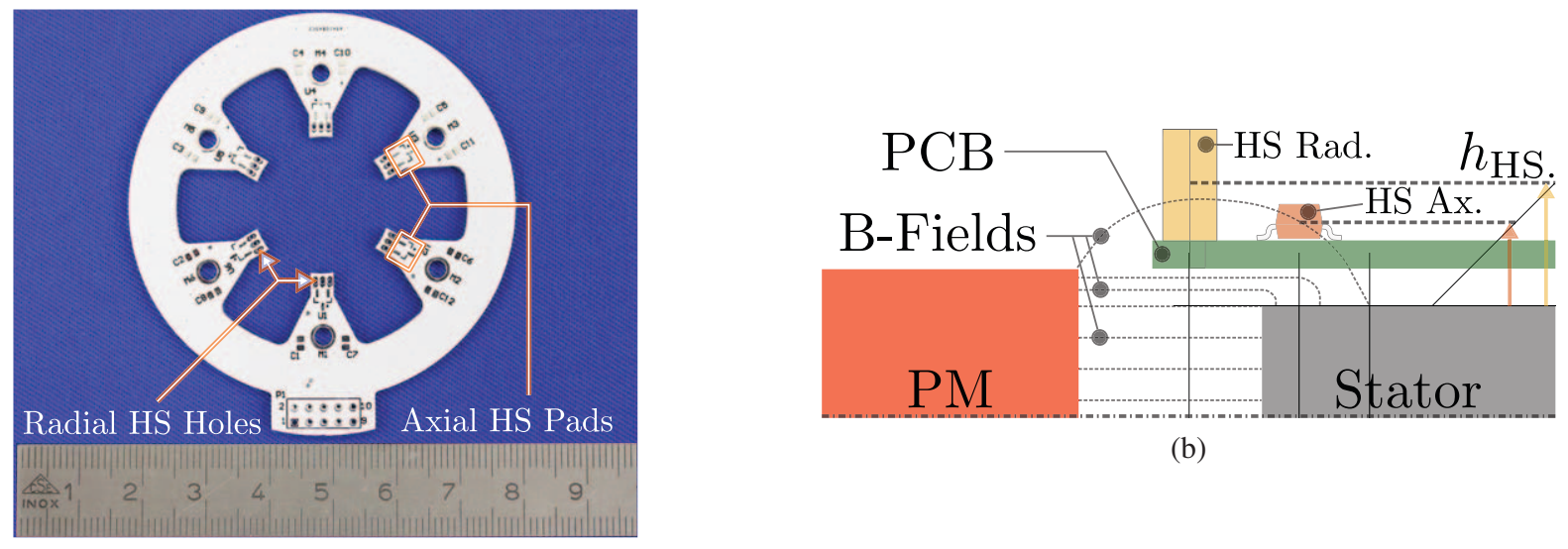

(b)

(a)

Fig. 5: (a) PCB for the mounting of 6 radial or axial Hall-effect sensors. (b) Cross-section of the halleffect sensor placement on the PCB, with vertical axial symmetry. The radial and axial sensors are fixed at radii $\left(r_{\mathrm{HS}}, h_{\mathrm{HS}}\right)=(13,8.3) \mathrm{mm}$ and $(16,7.3) \mathrm{mm}$, are depicted in yellow and orange, respectively. 

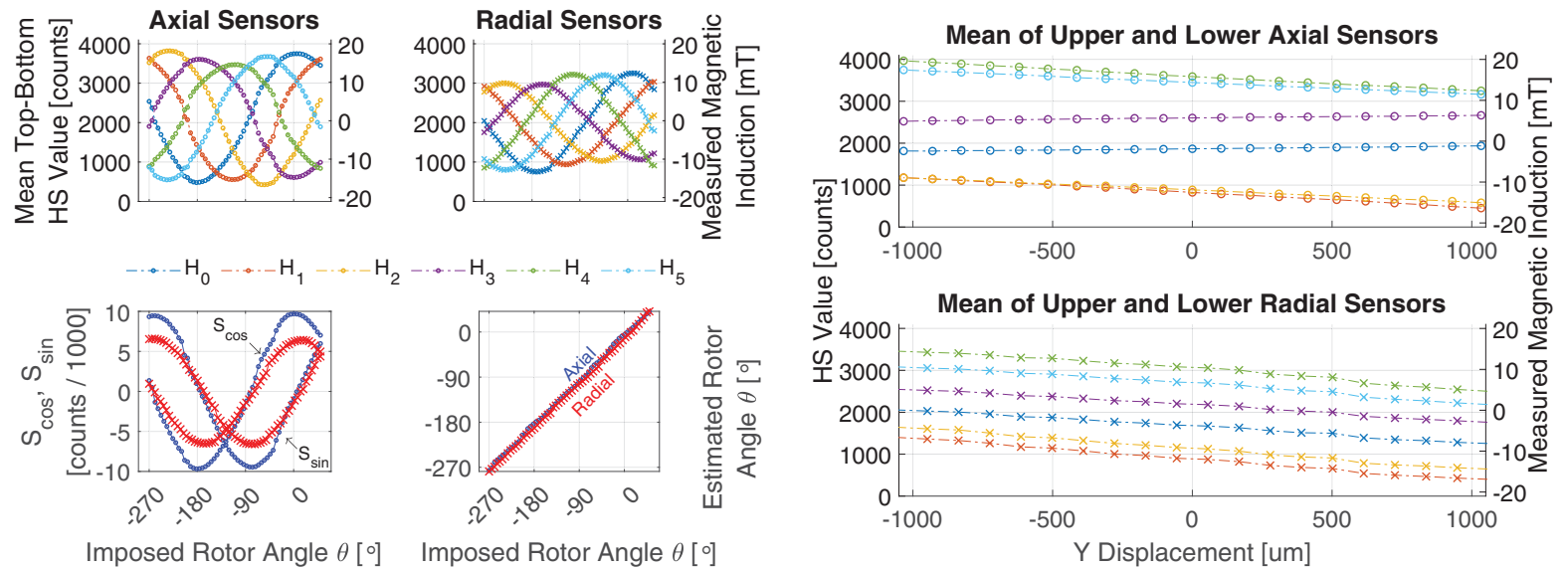

(b)

(a)

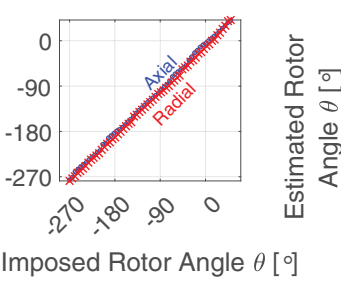

Fig. 6: Sensor values and angle estimation when (a) rotor is turned as $\theta=[-270: 5: 30]^{\circ}$ at the center of the airgap. (b) Sensor values with rotor displaced as in $y=[-1000: 100: 1000] \mu \mathrm{m}$ at fixed $\theta=270^{\circ}$.

\section{Validation of Rotor Radial Position Estimation}

Figure $6 \mathrm{~b}$ displays how the mean values of the bottom and top sensors change when the north pole of the rotor is between $\mathrm{H}_{4}$ and $\mathrm{H}_{5}$ and is displaced perpendicular to them, i.e. from $\mathrm{H}_{3}$ and $\mathrm{H}_{0}$.

In this case, two different behaviours are observed. The measured field of radial sensors $\mathrm{H}_{0}$ and $\mathrm{H}_{3}$ (perpendicular to the rotor poles) are affected by rotor displacement, and this is not foreseen by the model. In fact, the field gradients perpendiculars to the poles are as large as those next to the rotor poles, i.e. all sensors measure the same slope. Thus, parameter $\tau_{k}$ from Eq. (1) should not be neglected, so the proposed model estimates rotor position with lower accuracy.

Yet this is not the case of axial sensors. An quasi-null gradient is present in sensors $\mathrm{H}_{0}$ and $\mathrm{H}_{3}$, so $\tau_{k}$ can be neglected and the model holds valid. Axial sensors therefore enable a more accurate rotor position estimation.

With the north pole facing between $\mathrm{H}_{4}$ and $\mathrm{H}_{5}$, the position of the rotor is displaced in $x, y$ and $x-y$ direction. The hall-effect sensor values are recorded and the position on stator coordinates $\left(S_{\mathrm{X}_{\mathrm{st}}}, S_{\mathrm{Y}_{\mathrm{st}}}\right)$ is

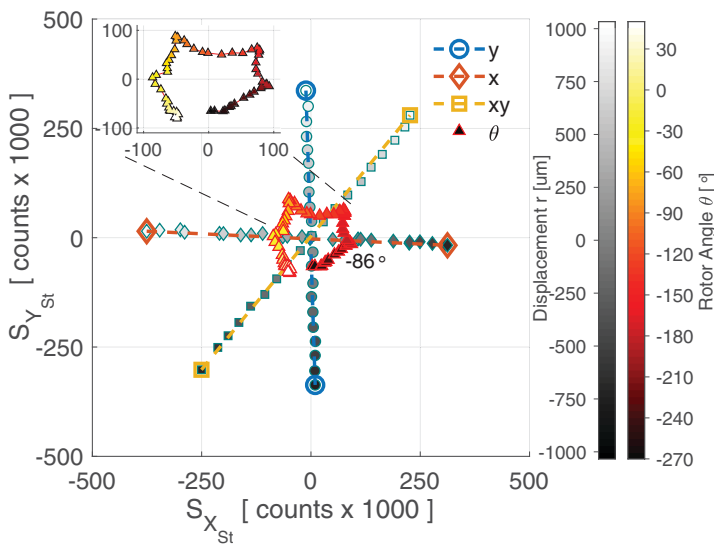

(a)

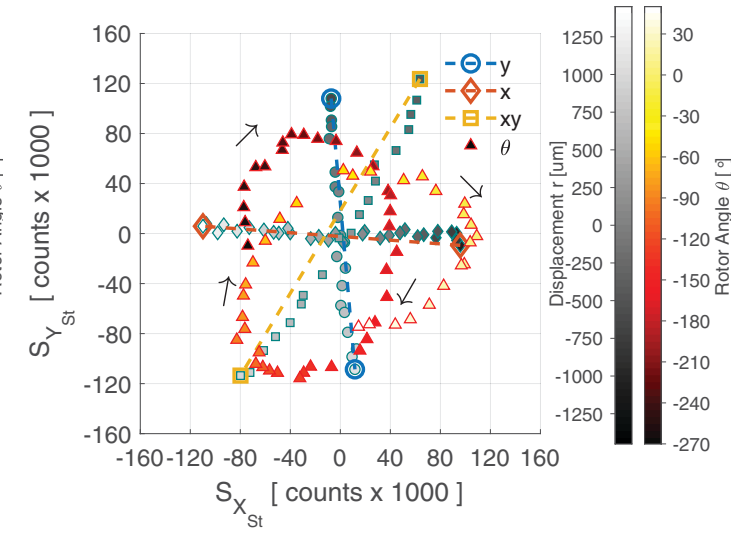

(b)

Fig. 7: Estimated rotor position $\left(S_{\mathrm{X}_{\mathrm{st}}}, S_{\mathrm{Y}_{\mathrm{st}}}\right)$ for a displacement (marked on the gray color bar) along the $x$, $y, x-y$ axes and half a turn of a centric rotor. The estimated position of the rotor turning in the middle of the airgap (see Fig. 6a) is marked with a $\triangle$, with rotor angle displayed on the red color bar. The position cloud for (a) axial and (b) radial Hall-effect sensors is shown. 
computed and displayed on Fig. 7. The estimated position of the rotor during the rotation of Fig. 6a is also plotted.

Figure $7 \mathrm{a}$ shows the estimated $\left(S_{\mathrm{X}_{\mathrm{st}}}, S_{\mathrm{Y}_{\mathrm{st}}}\right)$ using the axial sensors. The angle between the estimated positions is also depicted. Their non-perfect perpendicularity may be due to the a non-stiff enough test bench, so that the rotor deflects in $x$ direction, i.e. the direction of magnetization of the rotor during the test, due to the stark radial stiffness of the drive configuration.

The rotor is turned for $270^{\circ}$ and it is estimated that the rotor slightly budges. This hints once again that the test bench could slightly bend due to the radial strong stiffness of the drive configuration.

The experience is replicated using the radial sensors. The estimated positions of Fig. 7b on $x$ and $y$ are once again (almost) perpendicular, yet the signal amplitude is significantly reduced in comparison to the previous case.

Moreover, a second harmonic appears in the estimated position when the rotor is just turned in the middle of the airgap. The amplitude of the ripple is comparable to the amplitudes of the displacements as far away as $1 \mathrm{~mm}$, making this approach not suitable for accurate position estimation. Rotor eccentricity impacts the airgap flux distribution, and axial fields are probably less sensible to this dependency.

The signal amplitude $r \cdot s_{r}$ of Eq. (10) and its linearized sensitivity $s_{r}$ are plotted on Fig. 8 for axial and radial sensors. For the axial sensors, sensitivities $s_{r}$ in the $x, y$ and $x-y$ direction, and their differences could be due to non-perfect centering of the PCBs onto the drive, imperfect placement of the DRV5055s or test bench bending. They present only slight differences that can ultimately be corrected via software.

The sensitivity of the radially-placed sensors is approximately 3 times lower than that of the axial sensors. Moreover, it varies significantly depending on the direction of rotor displacement. This makes the radial configuration and its chosen measurement locus unsuitable for rotor position estimation purposes.

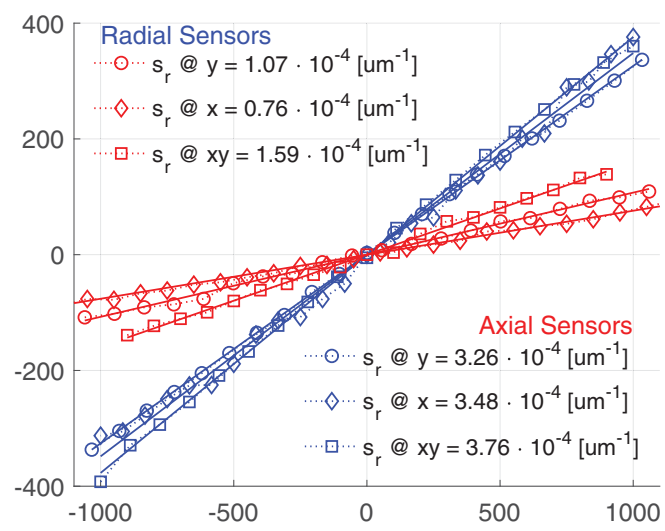

Fig. 8: Calculated radial rotor position sensitivities $s_{r}$ for axial and radial sensor mounting.

\section{Validation of Rotor Axial Position Estimation}

The validity of the axial rotor position estimation is tested in Fig. 9. As expected, the amplitude of the bottom sensors decreases, whereas that from the top sensors increases. The axial sensors respond in a more linear fashion to the $z$ displacement than its radial counterparts, which tend to be more parabolic towards $-2000 \mu \mathrm{m}$ and $2000 \mu \mathrm{m}$.

Signal $D_{z}$ and its corresponding sensitivity $s_{z}$ are linear and constant (respectively) throughout this $z$ range. Coincidently enough, both approaches render extremely similar $D_{z}$ trends and sensitivities $s_{z}$, with the axial approach being $0.6 \%$ more sensitive.

\section{Conclusion}

This paper analyses how the rotor $(x, y, z)$ position and its angular orientation $\theta$ can be obtained by measuring stray fields outside the airgap. This eases the encapsulation of a rotor, thus widening the ap- 


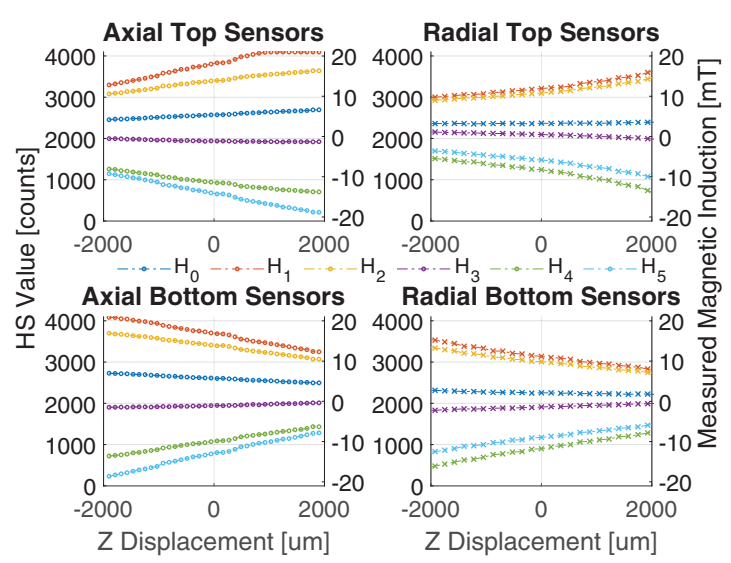

(a)

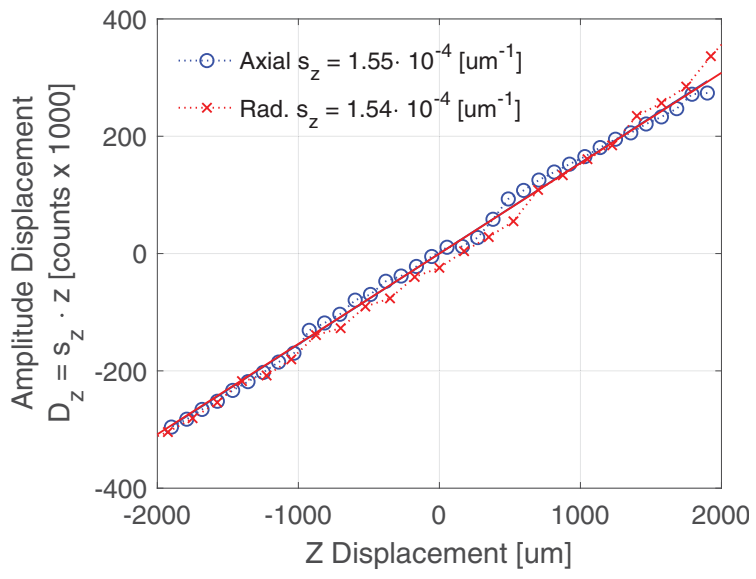

(b)

Fig. 9: (a) Measured hall-effect sensor values when rotor is displaced from $z=[-2000: 100: 2000] \mu \mathrm{m}$ with $(r, \theta)=\left(0 \mathrm{~mm}, 60^{\circ}\right)$ for axial and radial sensors and their (b) estimated axial rotor position signal $D_{z}$ and sensitivity $s_{z}$.

plication spectrum of bearingless disc drive. Rotor position estimation is thus enabled by off-the-market Hall-effect sensors which are simply mounted on a PCB onto the motor. With the chosen measurement placement, axial-field sensors render better results as their radial-field mounted versions.

A simplified model correlates the displacements of the rotor and the measured fields, and it is tested on a bench test. It enables measuring the angular and axial position of the rotor seamlessly, by either measuring the radial or axial field of the rotor. Nevertheless, the proposed model fits axial rotor magnetic fields better. Therefore, the radial rotor position was only accurately estimated by using axial sensors.

Radial magnetic sensing measured additional harmonics that do not correspond to the actual rotor position at their chosen measurement locus. A more complex modelling of the fields is necessary to clarify this behaviour and propose a better sensing scheme. Thus, this publication can still be enriched by rendering the mathematical model more complex to account for higher order harmonics and phase-shifts in the airgap and the estimation errors that they might cause.

Stray flux models could be applied so that the here-studied Hall-effect sensor position can be theoretically optimized on the design phase of the drive. Undesired harmonics or effects, such as those measured by the radial Hall-effect sensors, could be therefore minimized.

Further publications might also research the estimation of rotor position for a generalized number of Hall-effect sensors and any number of pole-pairs. Similarly, the model can be tested for slotted drives. More exhaustive measurements with a stiffer test bench must be taken into considerations. Test-bench imperfections could be clarified by measuring the real rotor displacement vs. its imposed displacement on the test bench.

Furthermore, the effect of motor excitation should be scrutinized. Drive or bearing currents on motor coils generate magnetic fields, which are superposed to those from the rotor and are thus measured by the sensors. The nature of this disturbance upon rotor position estimation, and eventual current compensation scheme can be further studied.

\section{References}

[1] N. Bianchi, S. Bolognani, and F. Luise, "Potentials and limits of high speed PM motors," 38th IAS Annual Meeting on Conference Record of the Industry Applications Conference, 2003., vol. 3.

[2] A. Borisavljevic, Limits, Modeling and Design of High-Speed Permanent Magnet Machines. Springer Theses, Berlin, Heidelberg: Springer Berlin Heidelberg, 2013. 
[3] R. Larsonneur, Design and control of active magnetic bearing systems for high speed rotation. $\mathrm{PhD}$ thesis, ETH Zürich, 1990.

[4] I. Takahashi, T. Koganezawa, G. Su, and K. Ohyama, "A super high speed PM motor drive system by a quasi-current source inverter," IEEE Transactions on Industry Applications, vol. 30, pp. 683690, May 1994.

[5] M. H. Kimman, Design of a Micro Milling Setup with an Active Magnetic Bearing Spindle. PhD thesis, TU Delft, 2010.

[6] B. Klammer, A. Zochbauer, H. Mitterhofer, and W. Gruber, "Topology Comparison and Design of a Slotted Bearingless High-Speed Permanent Magnetic Synchronous Machine," in 2019 IEEE International Electric Machines Drives Conference (IEMDC), pp. 1-8, May 2019.

[7] J. Asama, D. Suzuki, T. Oiwa, and A. Chiba, "Development of a Homo-Polar Bearingless Motor with Concentrated Winding for High Speed Applications," in 2018 International Power Electronics Conference (IPEC-Niigata 2018 -ECCE Asia), pp. 157-160, May 2018.

[8] Schweitzer, Gerhard and E. H. Maslen, Magnetic Bearings. 2009.

[9] K. Li, G. Cheng, X. Sun, D. Zhao, and Z. Yang, "Direct Torque and Suspension Force Control for Bearingless Induction Motors Based on Active Disturbance Rejection Control Scheme," IEEE Access, vol. 7, pp. 86989-87001, 2019.

[10] M. Schuck, A. D. S. Fernandes, D. Steinert, and J. W. Kolar, "A high speed millimeter-scale slotless bearlngless slice motor," in 2017 IEEE International Electric Machines and Drives Conference.

[11] M. Noh, W. Gruber, and D. L. Trumper, "Low-cost Eddy-current Position Sensing for Bearingless Motor Suspension Control," in 2017 IEEE International Electric Machines and Drives Conference.

[12] B. Warberger, T. Reichert, T. Nussbaumer, and J. W. Kolar, "Design considerations of a bearingless motor for high-purity mixing applications," in SPEEDAM 2010, pp. 1454-1459, 2010.

[13] H. Bleuler, H. Kawakatsu, W. Tang, W. Hsieh, D. K. Miu, Y. C. Tai, F. Moesner, and M. Rohner, "Micromachined Active Magnetic Bearings," 4th International Symposium on Magnetic Bearings, no. August, pp. 349-352, 1994.

[14] V. Kluyskens, C. Dumont, and B. Dehez, "Description of an Electrodynamic Self-Bearing Permanent Magnet Machine," IEEE Transactions on Magnetics, vol. 53, no. 1, pp. 1-9, 2017.

[15] M. Miyoshi, H. Sugimoto, and A. Chiba, "Axial vibration suppression by field flux regulation in two-axis actively positioned permanent magnet bearingless motors with axial position estimation," Proceedings - 2016 22nd International Conference on Electrical Machines, ICEM 2016, vol. 54.

[16] T. Instruments, "DRV5055 High accuracy 3.3 V or 5 V ratiometric bipolar Hall-effect sensor."

[17] E. Magnetics, "Rare Earth Neodymium NdFeB Magnets Datasheet," 2014.

[18] Metglas, "PowerLite C-Cores Technical Bulletin Alloy 2605SA1." 